\title{
The ways of finding uncountable set solutions for equations of
}

$$
p A^{a} \pm q B^{b} \equiv r D^{c} \text {. }
$$

(elementary aspect)

\section{PROF. DR. K. RAJA RAMA GANDHI ${ }^{1}$, REUVEN TINT ${ }^{1}$, MICHAEL TINT $^{2}$}

Resource person in Math for Oxford University Press and Professor at BITS-Vizag ${ }^{1}$

Number Theorist, Israel ${ }^{1}$

Software Engineer, Israel ${ }^{2}$

Email: editor126@gmail.com, reuven.tint@gmail.com, tintmisha@gmail.com

Abstract. Now we shall give the variants of uncountable set solutions that are a prime numbers and also a not coprime numbers of

$$
p A^{a} \pm q B^{b} \equiv r D^{c}
$$

, as well as, the version of anti-solution for the Pillai's conjecture.

1.1. Algorithm for finding uncountable set solutions in positive integers of the equation

$$
u^{a_{1}} A^{a}+\vartheta^{b_{1}} B^{b} \equiv r^{c_{1}} D^{c}[\mathbf{1}]
$$

for arbitrary of pairwise coprime $a, b, c$ and arbitrary natural $a_{1}, b_{1}, c_{1}, u, \vartheta, r$.

1.1.1. We have the identity

$$
u_{0}\left(\vartheta_{0}-r_{0}\right)+\vartheta_{0}\left(r_{0}-u_{0}\right) \equiv r_{0}\left(\vartheta_{0}-u_{0}\right) \quad[2]
$$

where $u_{0}, \vartheta_{0}, r_{0}-$ are arbitrary coprime $\left(u_{0}, \vartheta_{0}, r_{0}\right)=1$

natural numbers, such that

and

$$
u_{0}=u^{a_{1}} ; \quad \vartheta_{0}=\vartheta^{b_{1}} ; r_{0}=r^{c_{1}}
$$

$$
\begin{gathered}
\vartheta^{b_{1}}>r^{c_{1}} ; r^{c_{1}}>u^{a_{1}}[\mathbf{3}] \\
\left(x=\vartheta^{b_{1}}-r^{c_{1}}\right)+\left(y=r^{c_{1}}-u^{a_{1}}\right) \equiv\left(z=\vartheta^{b_{1}}-u^{a_{1}}\right) \\
u^{a_{1}}\left(\vartheta^{b_{1}}-r^{c_{1}}\right)+\vartheta^{b_{1}}\left(r^{c_{1}}-u^{a_{1}}\right) \equiv \\
\equiv r^{c_{1}}\left(\vartheta^{b_{1}}-u^{a_{1}}\right)
\end{gathered}
$$

1.1.2 . In addition, we have identity

$$
\begin{gathered}
{\left[A^{a}=\left(x^{\alpha} y^{q c} z^{m b}\right)^{a}\right]+\left[B^{b}=\left(x^{p c} y^{\beta} z^{m a}\right)^{b}\right] \equiv} \\
\equiv\left[D^{c}=\left(x^{p b} y^{q a} z^{\gamma}\right)^{c}\right]
\end{gathered}
$$

if $x+y=z$.

The values of the parameters of all the exponents found from the equations:

$$
\begin{gathered}
\alpha a-p b c=1 \\
\beta b-q a c=1 \\
\gamma c-m a b=1
\end{gathered}
$$


All infinite set of solutions of these equations, supplemented by one

$$
(a=b, c)=1 \text { and }
$$

$$
x+y=z,
$$

where $x, y$-are arbitrary natural numbers, give solutions of the equations [1] are not coprime:

It follows that,

$$
u^{a_{1}} A^{a}+\vartheta^{b_{1}} B^{b} \equiv r^{c_{1}} D^{c}[\mathbf{5}],
$$

1.2.2. If

$$
u_{0}=u^{n} ; \vartheta_{0}=\vartheta^{n} ; r_{0}=r^{n}
$$

where $n-$ is arbitrary natural number,

$$
\begin{gathered}
u^{n}\left(\vartheta^{n}-r^{n}\right)+\vartheta^{n}\left(r^{n}-u^{n}\right) \equiv r^{n}\left(\vartheta^{n}-u^{n}\right), \\
\left(x_{1}=\vartheta^{n}-r^{n}\right)+\left(y_{1}=r^{n}-u^{n}\right) \equiv\left(z_{1}=\vartheta^{n}-u^{n}\right), \\
A_{1}=x_{1}^{\alpha} y_{1}^{g c} z_{1}^{m b} ; B_{1}=x_{1}^{p c} y_{1}^{\beta} z_{1}^{m a} ; \\
D_{1}=x_{1}^{p b} y_{1}^{q a} z_{1}^{\gamma}
\end{gathered}
$$

$\left(x_{1}+y_{1}=z_{1}\right)$, then

$$
u^{n} A_{1}^{a}+\vartheta^{n} B_{1}^{b} \equiv r^{n} D_{1}^{c} \quad[6]
$$

1.2.3. Example.

1)

$$
\left(x_{1}^{\alpha} y_{1}^{q c} z_{1}^{m b}\right)^{a}+\left(x_{1}^{p c} y_{1}^{\beta} z_{1}^{m a}\right)^{b} \equiv\left(x_{1}^{p b} y_{1}^{q a} z_{1}^{\gamma}\right)^{c} .
$$

Suppose,

Then,

$$
a=4 ; b=5 ; c=7 ;(4,5,7)=1 \text {. }
$$

$$
\begin{gathered}
\alpha \times 4-p \times 5 \times 7=1 \\
p=1 ; \alpha=9 \\
\beta \times 5-q \times 4 \times 7=1 \\
q=3 ; \beta=17 . \\
\gamma \times 7-m \times 4 \times 5=1 \\
m=1 ; \gamma=3 \\
\left(x_{1}^{9} y_{1}^{21} z_{1}^{5}\right)^{4}+\left(x_{1}^{7} y_{1}^{17} z_{1}^{4}\right)^{5}=\left(x_{1}^{5} y_{1}^{12} z_{1}^{3}\right)^{7}
\end{gathered}
$$

,if

$$
x_{1}+y_{1}=z_{1}
$$


2) Similarly, $u=2 ; \vartheta=5 ; r=3, n=3$.

$$
\begin{gathered}
x_{1}=\vartheta^{n}-r^{n}=5^{3}-3^{3}=98 \\
y_{1}=r^{n}-u^{n}=3^{3}-2^{3}=19 \\
z_{1}=\vartheta^{n}-u^{n}=5^{3}-2^{3}=117 \\
2^{3}\left(98^{9} \times 19^{21} \times 117^{5}\right)^{4}+5^{3}\left(98^{7} \times 19^{17} \times 117^{4}\right)^{5}= \\
=3^{3}\left(98^{5} \times 19^{12} \times 117^{3}\right)^{7}
\end{gathered}
$$

3)

$$
\begin{gathered}
98^{35} \times 19^{84} \times 117^{20} \times\left(2^{3} \times 98+5^{3} \times 19\right)= \\
=98^{35} \times 19^{84} \times 117^{20} \times 3^{3} \times 117 \\
784+2375=3159
\end{gathered}
$$

4)

$$
98+19=117
$$

5)

$$
2^{3} \times 98+5^{3} \times 19=3^{3} \times 117
$$

P.S. Using the [3] it follows that

\section{1)}

2)

$$
\vartheta^{b_{1}}>r^{c_{1}} ; b_{1} \times \operatorname{Ln} \vartheta>c_{1} \operatorname{Ln} r \text { and } b_{1}>c_{1} \times \frac{\operatorname{Ln} r}{\operatorname{Ln} \vartheta}
$$

$$
r^{c_{1}}>u^{a_{1}} ; c_{1} \times \operatorname{Ln} r>a_{1} \operatorname{Ln} u \text { and } c_{1}>a_{1} \times \frac{\operatorname{Ln} u}{\operatorname{Ln} r}
$$

\section{$\S 2$}

\section{Version of anti-solution for Pillai's conjecture.}

2.1. Pillai's conjecture concerns: "For fixed positive integers $A, B, C$ the equation

$$
A x^{m}+B y^{n}=C
$$

has only finitely many solutions (x,y,m,n -are positive integers)". Anti-solution presented in other notations.

2.1.1. Let

$$
M x-N y=z[7]
$$

$M, N, x, y-$ are arbitrary natural numbers.

Then,

$$
M x^{\alpha a-p b}-N y^{\beta b-q a}=z[\mathbf{8}] .
$$


Here,

$$
\begin{aligned}
& \alpha a-p b=1(\alpha a, p b)=1 \\
& \beta b-q a=1(\beta b, q a)=1
\end{aligned}
$$

Multiplying [8] by

$$
t=x^{p b} y^{q a}
$$

we get

$$
M\left(x^{\alpha} y^{q}\right)^{a}-N\left(x^{p} y^{\beta}\right)^{b}=z x^{p b} y^{q a}[\mathbf{9}]
$$

2.1.2. We fix the parameters $M, N, x, y, q, a, p, \beta, b$. Indeed,

$$
\begin{gathered}
a=a_{1} \times a_{2} \times \ldots k \times \ldots a_{i}<\infty \\
b=b_{1} \times b_{2} \times \ldots t \times \ldots b_{j}<\infty
\end{gathered}
$$

2.1.3. The result is

$$
\begin{gathered}
M\left[\left(x^{\alpha} y^{q}\right)^{\prod_{i=1, i \neq k}^{i<\infty} a_{i}}\right]^{a_{k}}-N\left[\left(x^{p} y^{\beta}\right)^{\prod_{j=1 j \neq t}^{j<\infty} b j}\right]^{b_{t}} \equiv \\
\equiv z x^{p b} y^{q a}[\mathbf{1 0}], \\
1 \leq k<\infty ; 1 \leq t<\infty
\end{gathered}
$$

2.1.4. Therefore, from $[\mathbf{1 0}]$ it follows that, equation

$$
A x^{m}-B y^{n}=c
$$

has an infinite set of solutions for given values

, fixed value

$$
A=M ; B=N
$$

$$
C=z x^{p b} y^{q a}
$$

and arbitrary $\left(m=a_{i}, n=b_{j}\right)=1$.

Defined in this case " $C$ " does not always coincide with the fixed values, but may coincide with the fixed set.

\section{§3}

3.1.

Variants of finding uncountable set coprime solutions (all in each case) equations

(elementary aspect)

$$
m A^{a} \pm q B^{b}=r D^{c}
$$

3.1.1. With respect to

$$
B^{b} \pm A^{a}=D^{2}
$$

$$
\left(2^{3}+1=3^{2} ; 3^{4}-2^{5}=7^{2} ; 2^{9}-7^{3}=13^{2} ;\right.
$$

$7^{3}-3^{5}=10^{2} ; 15^{3}+7^{4}=76^{2}$ and etc.

,then

$$
A^{a}\left(B^{b} k \pm N\right)-B^{b}\left(A^{a} k-N\right) \equiv N D^{2}
$$

3.1.2. If

$$
N=r D^{c-2}
$$

where $k, r$-are arbitrary natural numbers, including $1, c>2$, 


$$
A^{a}\left(B^{b} k \pm r D^{c-2}\right)-B^{b}\left(A^{a} k-r D^{c-2}\right) \equiv r D^{c}[\mathbf{1 1}] .
$$

Compare with the identity

$$
x^{a}\left(y^{b} k-z^{c}\right)+y^{b}\left(z^{c}-x^{a} k\right) \equiv z^{c}\left(y^{b}-x^{a}\right)
$$

,where $x, y, z, a, b, c, k$-are arbitrary positive integers, including 1 .

Example:

$$
\begin{gathered}
15^{3}+7^{4}=76^{2} \\
B^{b}=15^{3} ; A^{a}=7^{4} ; D^{2}=76^{2} \\
k=2 ; c=3 ; r=3 .
\end{gathered}
$$

Therefore,

$$
\begin{gathered}
7^{4} \times\left(15^{3} \times 2+3 \times 76\right)-15^{3}\left(7^{4} \times 2-3 \times 76\right)=3 \times 76^{3} . \\
7^{4} \times 6978+15^{3} \times 4574=3 \times 76^{3}
\end{gathered}
$$

3.1.3. Let using [11]

$$
B^{b} k+r D^{c-2}=P[\mathbf{1 2}]
$$

,where $(B, D)=1$-are coprime,

$P-$ is arbitrary positive integer.

Then, all the solutions [12] give

$$
\begin{aligned}
& k=P\left(B^{b}\right)^{\varphi\left(D^{c-2}\right)-1}+D^{c-2} t \\
& r=p \frac{1-\left(B^{b}\right)^{\varphi\left(D^{c-2}\right)}}{D^{c-2}}-B^{b} t,
\end{aligned}
$$

where $\varphi\left(D^{c-2}\right)-$ is Euler function, equal to the number of positive integers coprime to $D^{c-2}$ and less $D^{c-2}$;

$t$-is any integer

Then,

$$
\begin{gathered}
k=2\left(15^{3}\right)^{\varphi(76)-1}+76 \times 3 \\
r=2 \times \frac{1-\left(15^{3}\right)^{\varphi(76)}}{76}-15^{3} \times 3 \\
\varphi(76)=\varphi\left(2^{2} \times 19\right)=\left(2^{2}-2\right)(19-1)=36 . \\
\varphi(76)=\varphi(37)=36 .
\end{gathered}
$$

Under the condition of

$$
10^{3} \div 37
$$


gives a remainder 1,36 can be replaced by a factor equal to 3 . Therefore, if $p=2$ then

$$
15^{3}\left[2 \times\left(15^{3}\right)^{3-1}+76 \times 3\right]+76 \times\left[2 \times \frac{1-\left(15^{3}\right)^{3}}{76}-15^{3} \times 3\right]=2 .
$$

3.1.3.1. If

$$
\begin{gathered}
P=A^{n-a} m \\
m \times A^{n}+q B^{b}=r D^{c}
\end{gathered}
$$

,where $n>a, c>2, m$ are arbitrary positive integers.

Thus we have,

$$
m \times 7^{n}+q \times 15^{3}=r 76^{c}
$$

, where as an example,

$q=7^{4} k-r 76^{c-2} ;$ and etc.

\section{References:}

[1] SOME ANTI-SOLUTIONS OF THE PILLAI'S CONJECTURE AND PROOF OF FERMAT'S LAST THEOREM. Bulletin of Mathematical Sciences \& Applications ISSN: 2278-9634 Vol. 2 No. 4 (2013), p. 47-64 p. 1, § 1, i.1.1. 